$\mathrm{FN}-377$

7550.623

DEVELOPMENT OF A REMOTE COMPUTER WORK STATION AT FERMILAB

Greg A. Chartrand

Fermi National Accelerator Laboratory, Batavia, Illinois 60510

$$
\text { and }
$$

W. Peter Trower

Physics Department, Virginia Polytechnic Institute and State University Blacksburg, Virginia 24061

December 1982 


\title{
DEVELOPMENT OF A REMOTE COMPUTER WORK STATION AT FERMILAB
}

\author{
Greg A. Chartrand \\ Computing Department \\ Fermi National Accelerator Laboratory \\ Batavia, Illinois 60510 \\ W. Peter Trower \\ Physics Department \\ Virginia Polytechnic Institute and State University \\ Blacksburg, Virginia 24061
}

\begin{abstract}
We describe a leased phone line linked terminal cluster designed to meet the experiment related computing needs of a remote user group. The functional and fiscal details of this arrangement are discussed.
\end{abstract}




\section{INTRODUCTION}

Virginia Tech, collaborating on Multiparticle spectrometer experiments 580 and 623 run in the Fermilab Meson Laboratory, wished to emulate our on-site use of the Fermilab computer resources from a remote work station at our home institution. In Fall 1980 we tested dial-up access using acoustical coupled voice-grade phone lines. We found this was prohibitively expensive and operationally unreliable. However, with Fermilab's encouragement we developed a terminal cluster built around a statistical multiplexor which were then being introduced on-site.

The configuration described here, evolved over the last two years, provides us a cost effective way to collaborate on experiments and at the same time, more fully utilize Fermilab's computing resources. 


\section{THE FERMILAB COMPUTER/COMMUNICATIONS SYSTEM}

Fermilab maintains a variety of computer systems accessible by both interactive and batch terminals. The Central computer Facility, CCF, primarily services the scientific analysis computing needs of user groups along with the laboratory staff. Here, three interconnected control Data CYBER 175 processors, each with 256K words of direct access memory, share 500K words of extended core storage, 12 Gbytes of disk storage, and 29 magnetic tape drives. Communication with the CCF is acomplished through two CDC 2551 Network Processor Units, NPU's, which provide 160 asynchronous user ports. The operating system is a multi-mainframe, common $I / 0$ queued version of the Network Operating System, NOS.

Computers for data acquisition are provided directly to experiments and development projects by dedicated Digital Equipment Corporation systems. Currently, the computing needs of an individual experiment are typically met by a PDP-11/45 which can typically have as much as $124 \mathrm{~K}$ words of memory, 5 Mbytes of disk storage and and multiple magnetic tape drives operating at up to 6250 BPI 125 IPS. In the future the larger data acquisitions computers will probably be replaced by systems with correspondingly enhanced capabilities.

Communication within the network, shown in Fig. 1, is provided by a port Selector (Micom 600) which acts as a data PABX allowing selective access to any of the computer systems by appropriate users. Currently the network supports 9 computer systems with 40 dial-in remote access modems of 3 types: Bell 113, Bell 212, and Vadic 3400. The network is accessed interactively on-site through 350 hard-wired video-keyboard terminals, many of them with graphics capabilities. 
Terminal clusters are implemented using Micom Micro series 800 statistical multiplexors with long or short haul modems, depending on the distances involved. Thus, several possible terminal connections with automatic error checking and correction, essential for reliable graphics and printer output, are provided over a single 4-wire phone circuit.

Figure 2 shows a typical cluster used with the data logging PDP-11 at the MPS, M6 beam line, used by experiment 623 . Here the 12-channel multiplexor communicates with its counterpart through 9600 baud 1 imited-distance modems operating on a 4-wire leased phone circuit. The terminal is connected to the PDP-11 by two asynchronous line-drivers over twisted wire pairs passing through a control box. The box allows three selectable operational modes: "COC" mode provides access to any computer on the network; "LOCAL" connects to the data acquisition, PDP-11, computer; and "REMOTE" connects the data acquisition computer to the port selector line allowing the PDP-11 to be used from any terminal on the network. With the latter arrangement, required remote program development and/or system troubleshooting can be acomplished on the data acquisition computer with a minimum of physical intervention by untrained persons who happen to be at the MPS portacamp. 
III. THE VIRGINIA TECH WORK STATION

The Virginia Tech work station, shown in Fig. 3 , is instrumented to support al1 aspects of our groups experiment related computing. It is configured to allow for maximum utilization of equipment and so several communications options are switch selectable.

Basic communication from Fermilab to Virginia Tech is handled by two pair of ancient, but reliable, Racal 5500/96 modems; 9600 baud primary, and 4800 baud backup. These modems are connected by a leased 3002 4-wire voice-grade phone line. Two Micom Micro 828/SAT 8-channel statistical multiplexors with satellite options provide the virtual terminal connections to Fermilab. Thus, several terminal and/or modem combinations can be selected.

The first three multiplexor channels provide graphics capabilities at 1200 baud. A Lear Siegler ADM-3 terminal with a Digital Engineering Retro-Graphics RG-512 graphics board and compatible GraphX-Cursor X-12 interactive joystick is on the first channel. The second is occupied by an ADM-3A+ with Digital Engineering GEN II RG-1000/LS-60 graphics. A Data Media Colorscan $10 \mathrm{H}$ terminal with Digital Engineering DM 800 graphics will provide a $640 \times 400$ resolution color display on channel 3. Each terminal has a switch selected connection from its output port to a Digital Equipment LA-120 printer enhanced with a Textprint DECLOR modification which allows black and white or color graphics as well as conventional text to be produced. From the channel 1 terminal, a Vadic 3455 modem can be switch selected, providing network independent dial-up access to non-Fermilab computer systems.

The fourth channel, operating at 2400 baud, will support a second LA-120 printer connected to a Fermilab Terminal Cluster Facility, TCF, port. This serial RS-232 line will make it possible to route long outputs to the printer. 
Channels 5 and 6 run ADM-3A+ terminals at 1200 baud and are used for program development, interactive debugging and text editing.

A Xerox-Diablo 630 ECS printer operated at 300 baud, used almost exclusively for text production, will soon replace the present 1650 on channel 7.

A Vadic VA-103 answer-only modem running at 300 baud, accessed remotely via telephone dial-in, operates on channel 8.

The option of using the phone in voice mode is provided by a switch. Remote access is usually accomplished on a Texas Instruments 745 which has upper-lower case character capability. Frequent use is made of this channel to access the MIT MACSYMA and the SLAC SPIRES systems through the Fermilab port selectors out-dialing capability. Virginia Tech provides all work station and communications equipment on both ends of the circuit. Whenever possible, the terminals and communications hardware selected are the same as those used at Fermilab. These choices reduced compatability problems and provide an equipment pool from which replacements can be drawn while repairs are being made. 


\section{CONCLUSIONS}

The Virginia Tech work station is currentiy used by two faculty, two post doctoral and one graduate student, and two technicians. The current total interactive use is $s 500$ hours per month.

The equipment replacement value in the Virginia Tech work station is $s$ $\$ 25 \mathrm{~K}$. The annual equipment upgrade costs are $s \$ 5 \mathrm{~K}$; repair costs are $s \$ 2 \mathrm{~K}$ as we have no maintanence contracts; and supplies run $s \$ 1 \mathrm{~K}$.

The telephone charges for the current year are $\backsim \$ 10 \mathrm{~K}$, increasing at a rate of $25 \%$ annual1y. Our rural location requires that we use the Bell system service which is not good, and whose the line quality is worse. We survive by the excellence of the Micom multiplexor alone.

Benefiting from the maturing of the network and the experience of Virginia Tech, 7 universities are now connected to Fermilab. This number is increasing at the rate of $\sim 3$ per year. Each has been able to satisfy his individual requirements in a cost-effective, reliable way.

Access to the data acquisition computer systems through remote terminal clusters in the experimental areas will provide the opportunity for al ternative means of development, system troubleshooting and file sharing. As new network features are added, the possible applications broaden. For example, if one multiplexor channel is connected to the local university computer, network access is provided to remote collaborators to share or transfer files. A switching arrangement like the one depicted in Fig. 2 would make such a connection selectable. 
ACKNOWLEDGEMENTS

We are pleased to acknowledge the essential contributions of R.E. Craven in procurement, testing, training, maintaining and documenting this system described here. The helpful, but always critical, encouragement which A.E. Brenner and his staff provided this project with the necessary conditions for its success. The work at Virginia Tech is supported in part by NSF grant PHY-80-20411. 


\section{References}

1. G. Chartrand, Mini-micro Systems June 1982, pp. 223-228.

\section{Figure Captions}

Figure 1. Schematic of Fermilab Computer Communications Network.

Figure 2. Schematic of Multiparticle Spectrometer Terminal Cluster. Figure 3. Schematic of Virginia Tech Work Station. 
JO 13TH FLOOR

VAX COPPUTER

4 WIRE GIRCUII
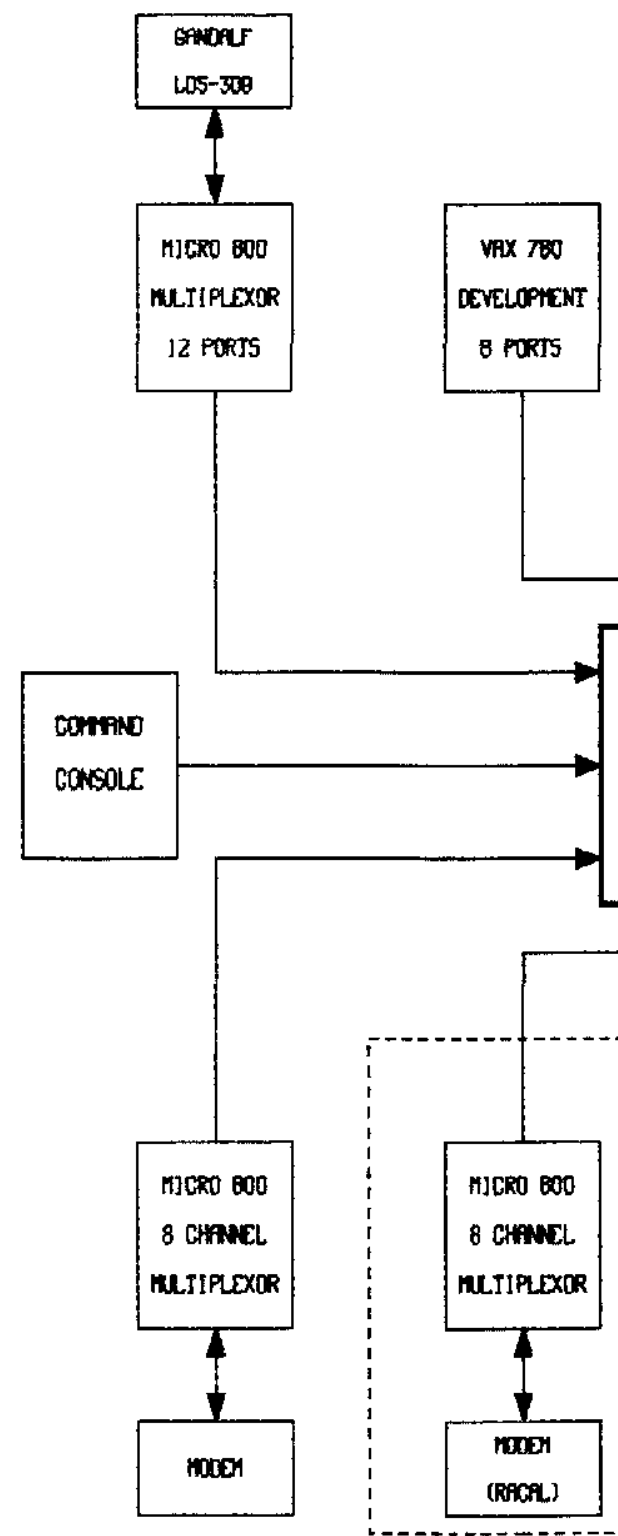

\section{FERMILAB DATA COMMUNICATIONS NETWORK}

TO CROSS

GRLLERY VAX

4 HIRE CIRCUIT

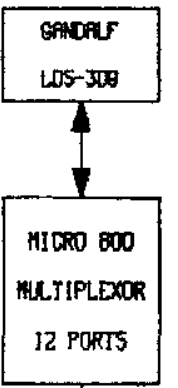

\begin{tabular}{|c|}
\hline 1.B.M. \\
4331 \\
8 PORTS \\
\hline
\end{tabular}

DEveropien

12 PBRTS
TE FERILA Do of TE VIREJN] JDXH

DATP MUIJPLXXR LIN

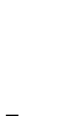




\section{VIRGINIA TECH REMOTE TERMINAL CLUSTER}

DIAL OUT

(V]RGINIA TECH,

StAC, ETC, J

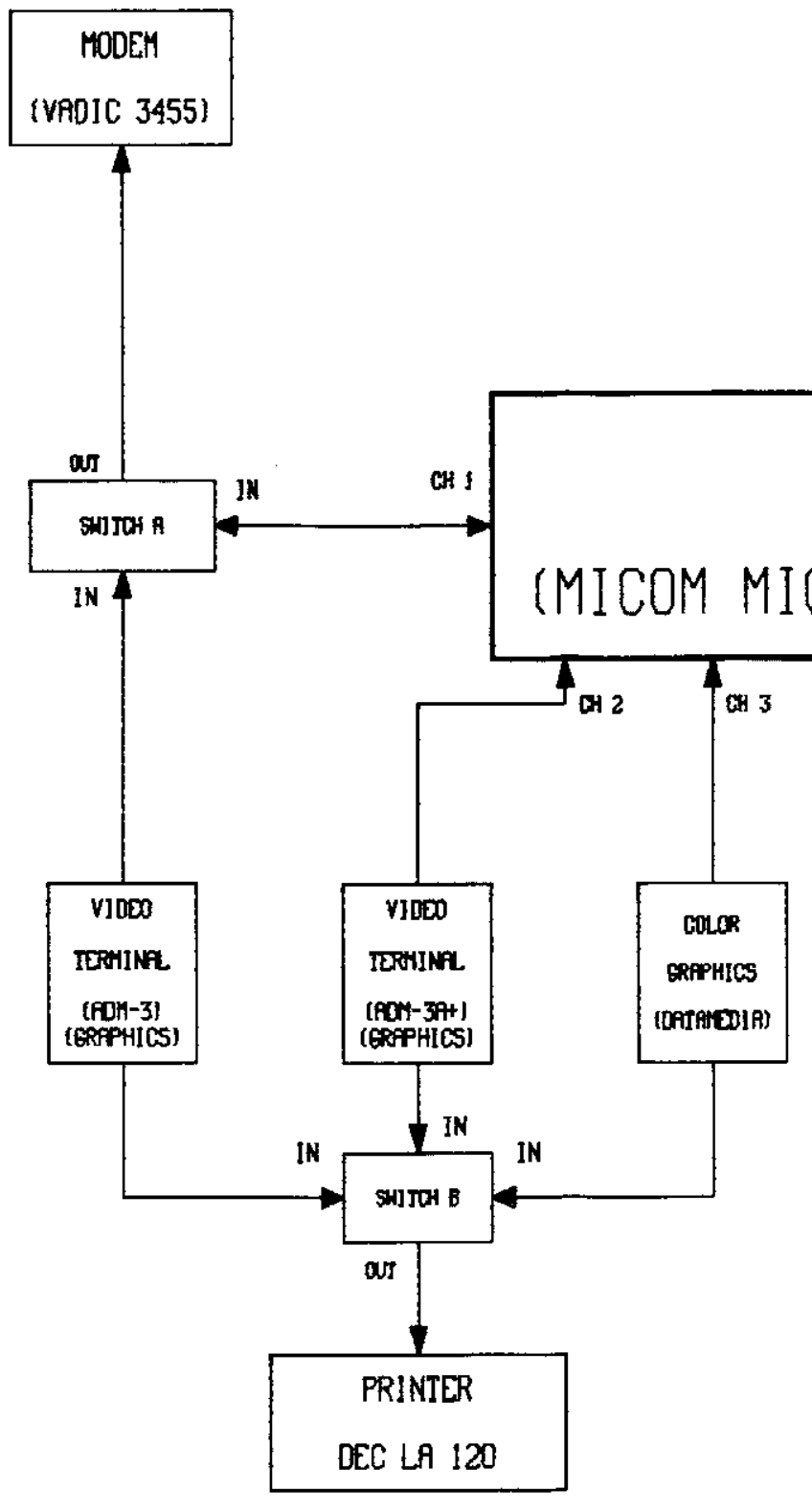

9600 BPLlD

SYNG COMPOSITE

LINE TO FERMILAB

\begin{tabular}{c|c|}
\hline MOOEM \\
RACPIL $5500 / 96$ \\
\hline
\end{tabular}
DIFL IN ACCESS TO FERMILAB VIA MULTIPLEXOR

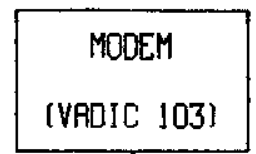


TO COMTFFL LABORATORY VIA 4 WIRE LEASED CIRCUIT
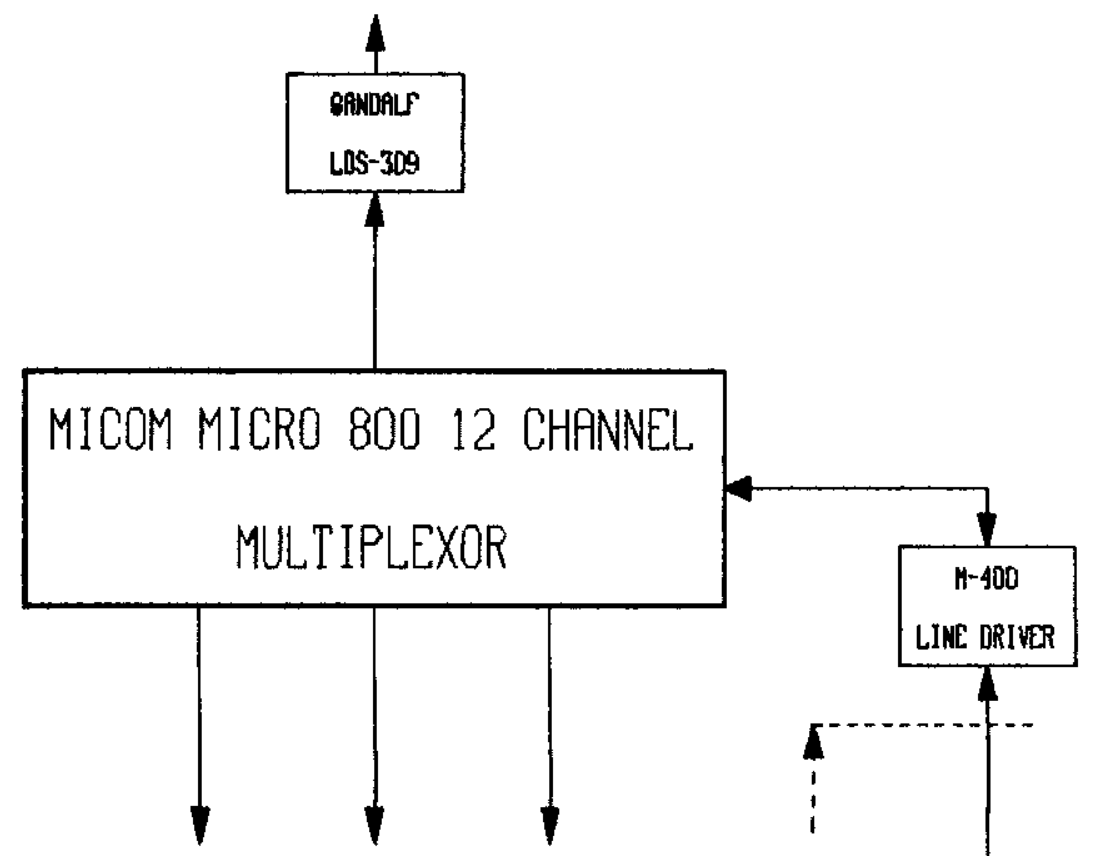

TO OTHER DATA RCOUISITION

APROX. I MJLE (HXX.)

COMPUTER SYSTEMS AND TERMINRLS

4 COND. CABQL

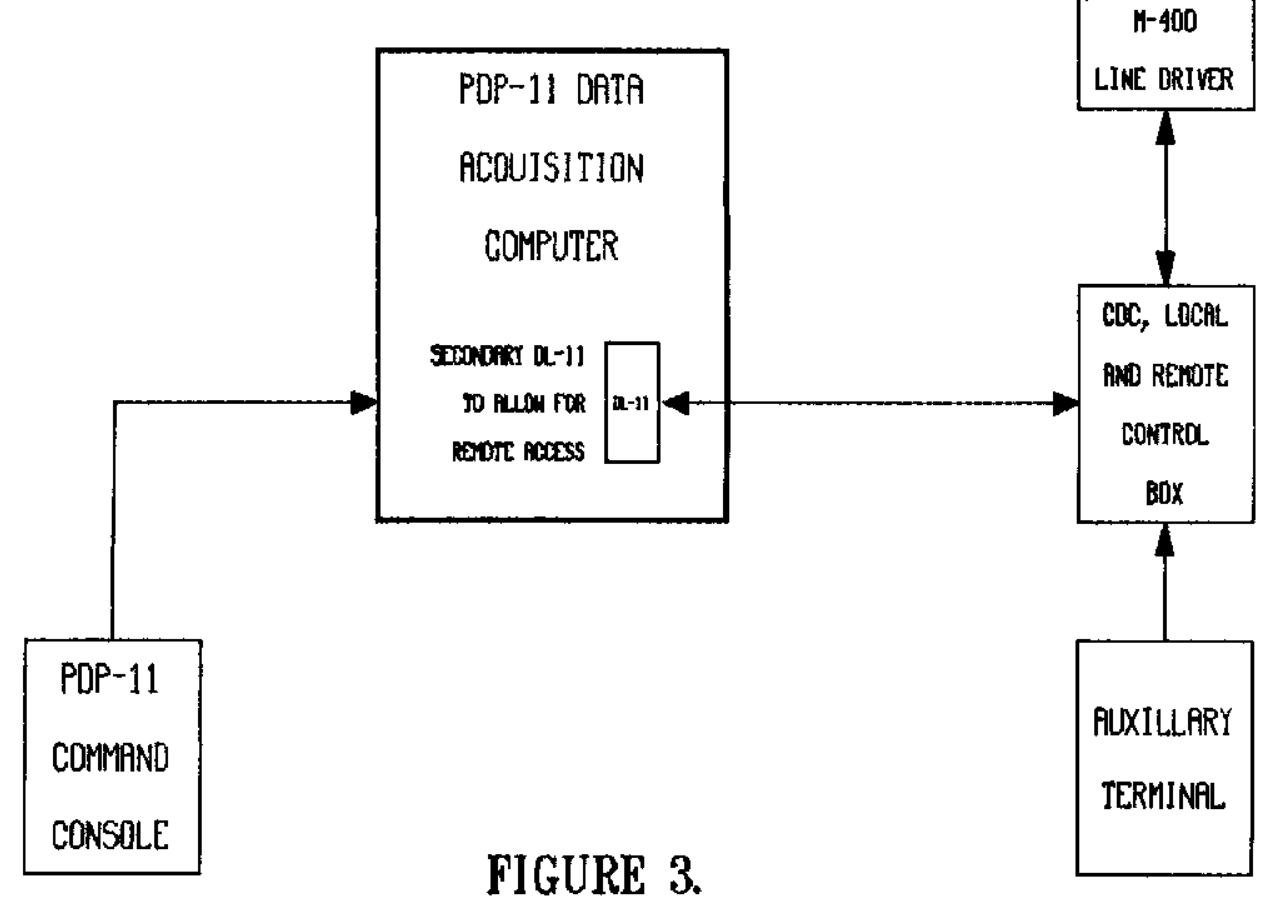

